Revue d'histoire de l'Amérique française

QREVUE D.HISTOIRE DE L'AMÉRIQUE FRANÇAISE

\title{
Pouvoir, religion et société en des temps indécis : Vincennes, 1763-1795
}

\section{Tangi Villerbu}

Volume 62, numéro 2, automne 2008

URI : https://id.erudit.org/iderudit/037522ar

DOI : https://doi.org/10.7202/037522ar

Aller au sommaire du numéro

\section{Éditeur(s)}

Institut d'histoire de l'Amérique française

\section{ISSN}

0035-2357 (imprimé)

1492-1383 (numérique)

Découvrir la revue

Citer cet article

Villerbu, T. (2008). Pouvoir, religion et société en des temps indécis : Vincennes, 1763-1795. Revue d'histoire de l'Amérique française, 62(2), 185-214.

https://doi.org/10.7202/037522ar

\section{Résumé de l'article}

Vincennes est une des communautés fondées par les Français à l'intérieur de l'Amérique du Nord qui demeure encore trop peu connue des historiens, surtout après 1763, lorsque le village devient possession britannique puis, rapidement, américaine. Il s’agit ici de jeter un éclairage neuf sur des temps troublés et incertains, entre la fin du Régime français et 1795, lorsque la puissance militaire amérindienne semble définitivement réduite dans la région et qu'un prêtre s'installe à demeure sous la double tutelle de l'État fédéral américain et l'évêque de Baltimore. Durant ces trente années, c'est le rapport entre les habitants et leurs autorités de tutelle qui est scruté : d'une part, la Couronne britannique, les États-Unis, et surtout dans les deux cas leurs représentants locaux, plus ou moins autonomes ; et d'autre part l'Église, qu'elle soit de Québec ou plus tard de Baltimore, et les prêtres qu'elle envoie sur place, difficilement et irrégulièrement. Les Vincennois, bien insérés dans un ensemble continental qui court de Québec à La Nouvelle-Orléans, de Baltimore à Saint-Louis, sont à la fois au fait des réalités les plus larges et prompts à défendre leur identité et leurs intérêts locaux, qu'ils soient d'ordre politique ou religieux. Cela doit se faire par des compromis, dans l'incompréhension souvent, sous la pression d'instances de contrôle extérieures que les Vincennois tentent de maîtriser eux-mêmes.
Tous droits réservés (C Institut d'histoire de l'Amérique française, 2009
Ce document est protégé par la loi sur le droit d'auteur. L’utilisation des services d'Érudit (y compris la reproduction) est assujettie à sa politique d'utilisation que vous pouvez consulter en ligne.

https://apropos.erudit.org/fr/usagers/politique-dutilisation/ 


\section{Pouvoir, religion et société en des temps indécis : Vincennes, I763-I795}

TANGI VILLERBU

Université de La Rochelle

RÉSUMÉ • Vincennes est une des communautés fondées par les Français à l'intérieur de l'Amérique du Nord qui demeure encore trop peu connue des historiens, surtout après 1763, lorsque le village devient possession britannique puis, rapidement, américaine. II s'agit ici de jeter un éclairage neuf sur des temps troublés et incertains, entre la fin du Régime français et 1795, lorsque la puissance militaire amérindienne semble définitivement réduite dans la région et qu'un prêtre s'installe à demeure sous la double tutelle de l'État fédéral américain et l'évêque de Baltimore. Durant ces trente années, c'est le rapport entre les habitants et leurs autorités de tutelle qui est scruté: d'une part, la Couronne britannique, les États-Unis, et surtout dans les deux cas leurs représentants locaux, plus ou moins autonomes; et d'autre part l'Église, qu'elle soit de Québec ou plus tard de Baltimore, et les prêtres qu'elle envoie sur place, difficilement et irrégulièrement. Les Vincennois, bien insérés dans un ensemble continental qui court de Québec à La Nouvelle-Orléans, de Baltimore à SaintLouis, sont à la fois au fait des réalités les plus larges et prompts à défendre leur identité et leurs intérêts locaux, qu'ils soient d'ordre politique ou religieux. Cela doit se faire par des compromis, dans l'incompréhension souvent, sous la pression d'instances de contrôle extérieures que les Vincennois tentent de maîtriser eux-mêmes.

ABSTRACT • Vincennes one of several French colonies founded in the interior of the North American continent. It has received very little attention from historians, especially for the period after 1763, when it passed, in quick succession, into the hands of the British and then the Americans. This article aims to shed new light on these troubled and uncertain times

1. La recherche présentée ici a bénéficié d'un travel research grant du Cushwa Center for the Study of American Catholicism de l'Université Notre Dame, Indiana. 
between the end of the French regime and 1795, when Amerindian military power seemed to be definitively weakened in the region and when a Catholic priest settled in Vincennes as both the federal Indian agent and the parish priest named by the bishop of Baltimore. This thirty-two year period saw a constant evolution in the relationship between the residents and local representatives of central authorities: first, there were the British Crown and the United States government, especially their local representatives who exercised a great deal of autonomy; secondly, there was the Catholic Church and the priests assigned to the local parish, with difficulty and irregularity, by the diocese of Quebec and later the diocese of Baltimore. The residents of Vincennes, well aware of their place within a vast North American network which ran from Quebec to New Orleans and from Baltimore to St. Louis, sought to defend their local identity and local interests, be they political or religious. Thus, residents attempted to become masters of their own destiny through compromise, despite frequent misunderstandings and under pressure from external authorities.

$\mathrm{V}^{\mathrm{s}}$ incennes a la malchance de se situer dans un entre-deux: entre le pays des Illinois au sens strict - les six villages des berges du Mississippi, Fort de Chartres, Prairie du Rocher, Saint Philippe, Sainte Geneviève, Kaskaskia, Cahokia - et Détroit, l'établissement de la Wabash n'a guère attiré les historiens. Ainsi l'histoire et la structure sociale du pays des Illinois ont été l'objet depuis une vingtaine d'années de nombreux travaux neufs et parfois contradictoires, certains y voyant des foyers quasi démocratiques comparables aux villages de la Nouvelle-Angleterre, d'autres au contraire des symboles de la permanence outre-Atlantique d'une hypothétique mentalité médiévale française, d'autres encore un ensemble de postes au profil complexe, entre grand commerce, esclavagisme et ferme tutelle coloniale ${ }^{2}$. Détroit de même a été revisité ${ }^{3}$, et même des postes

2. Le premier historien du Pays des Illinois fut Clarence W. Alvord avec The Illinois Country, 1673-1818 (Chicago, A.C. McClurg, 1920). Puis Margaret K. Brown, «La colonisation française de l'Illinois: une réévaluation ", Revue d'histoire de l'Amérique française, 39,4 (printemps 1986): 583-591; Winstanley Briggs, The Forgotten Colony: Le pays des Illinois, thèse de doctorat, University of Chicago, 1985) et «Le Pays des Illinois», William and Mary Quarterly, XLVII,1 (1990): 30-56; Carl J. Ekberg, Colonial Ste. Genevieve: An Adventure on the Mississippi Frontier (Gerald, Patrice Press, 1985), 551 p., French Roots in the Illinois Country: The Mississippi Frontier in the Colonial Times (Urbana, University of Illinois Press, 1998), 359 p. François Vallé and His World: Upper Louisiana Before Lewis and Clark (Columbia, University of Missouri Press, 2002), 316 p., Stealing Indian Women: Native Slavery in the Illinois Country (Urbana, University of Illinois Press, 2007), 236 p. Cécile Vidal, Les implantations françaises au Pays des Illinois (1699-1765), thèse de doctorat, EHESS, 1995 ; Robert K. Morissey, Bottomlands \& Borderlands: Empires and Identities in the $18^{\text {th }}$ Century Illinois Country, thèse de doctorat, Yale University, 2006.

3. Lina Gouger, Le peuplement colonisateur de Détroit, 1701-1765, thèse de doctorat, Université Laval, 2002 et «Migrer à Détroit au xvıII siècle : la part du monde rural dans le peuplement d'une zone frontière ", dans Gérard Bouchard, John A. Dickinson, Joseph Goy, dir., Les exclus de la terre en France et au Québec XVII $-\mathrm{XX} \mathrm{X}^{\mathrm{e}}$ siècles. La reproduction familiale dans la différence (Sillery, Septentrion, 
isolés comme Michillimackinac ${ }^{4}$ ou le Fort St. Joseph ${ }^{5}$ ont fait l'objet de nouvelles analyses, montrant la centralité de l'expérience française au cœur du continent pour appréhender l'histoire coloniale de l'ensemble de l'Amérique du Nord. Mais Vincennes est demeuré hors du regard des historiens, seul Carl J. Ekberg l'incluant dans ses travaux.

Et la fin du Régime français n'y change rien, au contraire, tant les communautés françaises, qui demeurent pourtant très dynamiques, en expansion constante pendant des décennies, semblent alors ne plus réellement intéresser les historiens francophones. Seuls semblent dignes d'intérêt les hommes des marges, ceux de la traite de la fourrure, employés des compagnies britanniques ${ }^{6}$ ou américaines ${ }^{7}$ qui ont permis l'émergence de communautés métisses ${ }^{8}$. Et les historiens américains, qui auraient dû

1998), 161-174. Marcel Bénéteau, dir., Passages: Three Centuries of Francophone Presence at le Détroit/ Le Passage du Détroit: 300 ans de présence francophone (Windsor, The Humanities Research Group, 2003), 348 p.

4. Susan Sleeper-Smith, " "[A]n Unpleasant Transaction on This Frontier” : Challenging Female Authority and Autonomy at Michillimackinac», Journal of the Early Republic, 25 (Fall 2005): 417-443; Keith Widder, "After the Conquest: Michillimackinac, A Borderland in Transition, 1760-1763 ", Michigan Historical Review, 34,1 (Spring 2008) : 43-62 ; Joseph L. Peyser et José António Brandão, dir., Edge of Empire: Documents of Michilimackinac, 1671-1716 (East Lansing, Michigan State University Press; Mackinac Island, Mackinac State Historic Parks, 2008), 224 p.

5. José António Brandão et Michael S. Nassaney, "A Capsule Social and Material History of Fort St. Joseph and Its Inhabitants (1691-1763)», French Colonial History, 7 (2006): 61-76 et «Suffering for Jesus: Penitential Practices at Fort St. Joseph (Niles, Michigan) During the French Regime», Catholic Historical Review, 94,3 (July 2008): 476-499.

6. Carolyn Podruchny, Making the Voyageur World: Travelers and Traders in the North American Fur Trade (Lincoln, University of Nebraska Press, 2006), 414 p. ; Robert Englebert, «Diverging Identities and Converging Interests : Corporate Competition, Desertion, and Voyageur Agency, 1815-1818», Manitoba History, 55 (June 2007): 18-24 et "Merchants Representatives and the French River World, 1763-1803 », Michigan Historical Review, 34,1 (Spring 2008) : 63-82.

7. William Swagerty, «Marriage and Settlement Patterns of Rocky Mountains Trappers and Traders », Western Historical Quarterly, 11,1 (Spring 1980): 159-180; Michael Lansing, "Plains Indian Women and Interracial Marriage in the Upper Missouri Trade, 1804-1868 ", Western Historical Quarterly, 31,4 (Winter 2000): 413-433; Nicole St-Onge, "The Persistence of Travel and Trade: St. Lawrence River Valley French Engagés and the American Fur Company, 1818-1840 ", Michigan Historical Review, 34,2 (Fall 2008): 17-38.

8. Sylvia Van Kirk, "Many Tender Ties»: Women in Fur-Trade Society in Western Canada, 1670-1870 (Winnipeg, Watson \& Dwyer, 1980), 303 p.; Jennifer S. H. Brown, Strangers in Blood: Fur Trade Company Families in Indian Country (Vancouver, University of British Columbia Press, 1980), 255 p. ; Jacqueline Peterson et Jennifer S. H. Brown, dir., The New Peoples : Being and Becoming Métis in North America (Lincoln, University of Nebraska Press, 1985), 266 p.; Lucy Eldersveld Murphy, A Gathering of Rivers: Indians, Métis, and Mining in the Western Great Lakes, 1737-1832 (Lincoln, University of Nebraska Press, 2000), 233 p. et «Public Mothers: Natives American and Métis Women As Creole Mediators in the Nineteenth-Century Midwest ", Journal of Women History, 14,4 (Winter 2003): 142-166; Melinda Marie Jetté, “We Have Almost Every Religion But Our Own” : French-Indian Communities Initiatives and Social Relations in Belle Prairie, Oregon, 1834-1837», Oregon Historical Quarterly, 108,2 (Summer 2007): 22-45. 
hériter du dossier, ne l'ont traité que marginalement. Le sort de Sainte Geneviève après la conquête est connu ${ }^{9}$, celui de Détroit entr'aperçu ${ }^{10}$ mais, par exemple, la naissance et l'existence de Prairie du Chien demeurent encore à étudier. Vincennes, en conséquence, avant comme après 1763 , constitue comme un point aveugle du continent. Ce n'est pas que l'historiographie de la région soit restée statique. À l'inverse, c'est en repensant l'histoire de la région des Grands Lacs depuis la colonisation française que l'histoire de l'Ouest, et celle de la conquête coloniale du continent par les États-Unis en général a été renouvelée en profondeur, par l'intermédiaire notamment de la notion de «middle ground» qui pour son inventeur Richard White recouvrait les espaces de rencontres entre peuples, cultures et empires qui menaient à des incompréhensions certes mais créatrices de nouvelles formes sociales culturelles. Or, pour White, Vincennes, "perhaps more than any other site in the Ohio Valley, captured the social, ethnic and political diversity of the Pays d'en Haut in the $1780{ }^{\prime 11}{ }^{11}$. Le village est alors exemplaire aussi, voire surtout, de la fin de ce «middle ground», lorsque les colons américains refusent de s'intégrer aux logiques préexistantes et de ce fait les détruisent en créant des frontières de toutes sortes là où elles n'existaient pas, en rigidifiant des rapports sociaux marqués auparavant par une certaine fluidité - qu'il ne faut ni surestimer ni mythifier pour autant. Ce nouveau récit nord-américain, enrichi, critiqué, élargi à d'autres régions que les Grands Lacs, est centré sur la relation entre les colons et les Amérindiens ${ }^{12}$. De ce fait, l'histoire

9. Susan C. Boyle, «Did She Generally Decide? Women in Ste. Geneviève, 1750-1805», William and Mary Quarterly, 44,4 (October 1987): 775-789; Walter A. Schroeder, Opening the Ozarks: A Historical Geography of Missouri's Ste. Genevieve District, 1760-1830 (Columbia, University of Missouri Press, 2002), 551 p. ; Bonnie Stepenoff, From French Community to Missouri Town: Ste. Genevieve in the Nineteenth Century (Columbia, University of Missouri Press, 2006), $232 \mathrm{p}$.

10. Jay Gitlin, «Freemasons and Speculators: Another Look at the Francophone Merchants of Detroit, 1796 to 1863 ", Humanities Research Group Working papers, Windsor University, 11 (2003): http://www.phaenex.uwindsor.ca/ojs/leddy/index.php/HRG/issue/view/53

11. Richard White, The Middle Ground: Indians, Empires and Republic in the Great Lakes Region, 1650-1815 (New York, Oxford University Press, 1991), 422.

12. Ibid., et Andrew R. L. Cayton et Fredrika J. Teute, dir., Contact Points : American Grontiers Grom the Mohawk Valley to the Mississippi, 1750-1830 (Chapel Hill, University of North Carolina Press, 1998), 391 p. ; Gilles Havard, Empire et métissages. Indiens et Français dans le Pays d'en haut, 1660-1715 (Québec/Paris, Septentrion/Presses de l'Université de Paris-Sorbonne, 2003), 858 p.; Alan Taylor, The Divided Ground: Indians, Settlers and the Northern Borderland of the American Revolution (New York, Alfred A. Knopf, 2006), 542 p.; Forum «The Middle Ground Revisited», Willam and Mary Quarterly, LXIII,1 (January 2006): 3-96. Les problématiques peuvent être élargies à l'ensemble du continent par le biais de concepts proches. Voir Jeremy Adelman et Stephen Aron, «From Borderlands to Borders: Empires, Nation-States, and the Peoples In-Between in NorthAmerican History ", American Historical Review, 104,3 (June 1999): 814-841; Theodore Binnema, 
des établissements stables des colons francophones ne s'en trouve guère enrichie, puisque le devant de la scène est occupé par les seules situations de contacts avec les Autochtones, ce qui, bien que fondamental dans la vie de ces communautés, ne recouvre pas, loin s'en faut, l'entièreté de leur expérience.

C'est sur ces bases neuves mais en recentrant le regard sur la communauté locale qu'il faut commencer à reconsidérer pleinement l'histoire de Vincennes. C'est-à-dire d'abord qu'il faut cesser d'y voir un foyer unique et isolé, mais au contraire une forme paradigmatique du palimpseste que fut l'histoire coloniale de la région, et plus largement encore de l'ensemble du continent en tant qu'il fut l'objet d'une conquête coloniale jusqu'au début du $\mathrm{xx}^{\mathrm{e}}$ siècle. Il faut ensuite considérer la colonisation comme une volonté de contrôle de la société, afin de la faire entrer en conformité avec des modèles coloniaux pensés dans les métropoles, une volonté donc de formation de frontières ethniques, culturelles, raciales tout autant que politiques. Une étude de Vincennes doit être l'analyse des différentes structures coloniales qui ont tenté de modeler et de remodeler sans cesse la société locale afin de la faire renter en conformité avec certains imaginaires "nationaux» ou religieux, comme des configurations adoptées localement en réponse ou bien souvent en parallèle à ces projets. Elle doit ainsi être l'étude des demandes des colons, de leur vision du monde et d'eux-mêmes au sein de ce monde: non pas seulement de leur réaction face à l'autorité, mais aussi de leurs prises d'initiatives, des négociations permanentes qu'ils mènent avec toutes les structures de pouvoir, car ils agissent eux-mêmes sur l'État comme sur l'Église ${ }^{13}$. Si contrôle social il y a, il ne peut qu'être le fruit de compromis.

Common and Contested Ground: A Human and Environmental History of the Northwestern Plains (Norman, University of Oklahoma Press, 2001), 263 p.; James F. Brooks, Captives and Cousins: Slavery, Kinship and Community in the Southwest Borderlands (Chapel Hill, University of North Carolina Press, 2002), 432 p.; Stephan Aron, American Confluence: The Missouri Frontier From Borderland to Border State (Bloomington, Indiana University Press, 2006), 301 p. ; Kathleen Duval, The Native Ground: Indians and Colonists in the Heart of the Continent (Philadelphia, University of Pennsylvania Press, 2006), 320 p. ; Juliana Barr, Peace Came in the Form of a Woman: Indians and Spaniards in the Texas Borderlands (Chapel Hill, University of North Carolina Press, 2007), $397 \mathrm{p}$.

13. René Hardy, Contrôle social et mutation de la culture religieuse au Québec, 1830-1930 (Montréal, Boréal, 1999), 284 p. et "Regards sur la construction de la culture catholique québécoise au XIX ${ }^{\mathrm{e}}$ siècle», Canadian Historical Review, 88,1 (March 2007): 7-40, qui présentent un large bilan historiographique de la question; Ollivier Hubert, Sur la terre comme au ciel: la gestion des rites par l'Église catholique du Québec (fin XVIII- $m i$-XIX $x^{\mathrm{e}}$ siècle) (Sainte-Foy, Les Presses de l’Université Laval, 2000), 341 p. La volonté romaine de prendre pied fermement sur le continent nord-américain à l'époque traitée ici est abordée par Luca Codignola, "Roman Catholic Conservatism in the New North Atlantic World, 1760-1829», William and Mary Quarterly, 64,4 (October 2007): 717-756. 
Les structures coloniales ici étudiées seront à la fois politiques et religieuses: il s'agit d'une part des autorités britanniques puis des colons et des autorités américaines, et d'autre part de l'Église catholique. Il s'agit en effet de prendre en quelque sorte l'histoire de Vincennes «en route», entre deux tournants de ce que François Furstenberg nomme la Longue Guerre, celle, indécise mais traitée par les historiens de manière trop souvent encore téléologique, menée pour le contrôle de l'Ouest transappalachien entre 1754 et $1815^{14}$.

En 1763, le Régime français prend fin, et Vincennes devient possession britannique, avant qu'en 1778 les forces américaines de George Rogers Clark n'envahissent la région. Des années d'instabilité chronique suivent, pendant lesquelles Vincennes et sa population encore très majoritairement francophone et catholique sont au centre d'un conflit à trois entre Britanniques, Américains et tribus indiennes (Piankashaws, Miamis) aux intérêts parfois divergents. Durant ces années, c'est encore le diocèse de Québec, jusqu'en 1787, qui possède la juridiction sur Vincennes, et qui tente, avec de faibles moyens, de contrôler la vie religieuse, et donc la vie sociale. Puis l'évêque de Baltimore, John Carroll, envoie son propre clergé sur place et tente une ferme reprise en main. En 1795, les Indiens signent le Traité de Greenville, qui les dépossède de la majeure partie de la vallée de l'Ohio et de ses affluents. L'histoire de Vincennes s'en trouve modifiée. Elle est faite pour l'essentiel d'incertitudes quant au statut de la communauté, aux identités religieuses, civiques, ethniques dont elle est porteuse, et quant à sa pérennité même parfois. C'est cette incertitude qui fait la richesse des questionnements de cette histoire des confins coloniaux de l'Amérique du Nord. Il ne sera pas question ici de faire une histoire sociale de Vincennes, une analyse de la question foncière ou de l'insertion de la communauté dans les mondes indiens. Tous ces éléments seront partie prenante d'une proposition modeste d'histoire culturelle des formes de l'incertitude coloniale, à l'échelle d'une communauté nord-américaine.

\section{FACE À LA VACANCE DES POUVOIRS, 1763-1778}

Dans une première phase, c'est Vincennes même, plus encore que les communautés voisines des rives du Mississippi, qui est menacé tant justement les instances de contrôle social semblent s'être totalement retirées de la région. L'intérêt des quelques sources éparses qui permettent d'in-

14. François Furstenberg, "The Significance of the Trans-Appalachian Frontier in American History", American Historical Review, 113 (June 2008) : 647-677. 
terroger le Vincennes des années 1763-1778 réside justement dans ce qu'elles révèlent de l'attitude des habitants face à cette vacance des pouvoirs civils et religieux. Elles permettent d'interroger les deux visions qui se sont opposées, jusqu'à maintenant souvent, à propos des Français ${ }^{15}$ de Vincennes: en avril 1772, le général Gage, en charge des nouvelles possessions britanniques, fait preuve d'une grande rudesse en décidant de vider Vincennes au motif que les Français y «mènent une vie vagabonde sans Gouvernement et sans Loi, interrompent le cours libre du Commerce, détruisent le Gibier et causent des Brouilleries infernales dans le Pais ${ }^{16}{ }^{\prime}$; c'était là s'inscrire en droite ligne dans la politique définie par la Proclamation Royale de 1763 : les terres à l'Ouest de Montréal étaient indiennes, la couronne ne pouvait y tolérer une population de colons qui parasitaient ses objectifs commerciaux et politiques et faisaient la preuve de la déchéance culturelle qui attendait le «civilisé » au contact du monde «sauvage».

Andrew Cayton, deux siècles plus tard, reprend finalement les mêmes arguments - développés précédemment pour Fort de Chartres par Winstanley Briggs ${ }^{17}$ - pour déclarer à l'inverse que les colons français sont comparables aux colons anglo-américains qui se révolteront en 1776, dans leur volonté d'autonomie à l'échelle de la communauté villageoise; il les rattache donc non plus à un imaginaire d'arriération mais bien de dynamisme et de modernité, à la fois commercialement ouverts et politiquement suffisamment matures pour une forme de démocratie locale ${ }^{18}$.

Vincennes fut fondé en 1731 et vécut sous le Régime français une triple vocation : militaire - c'est le fort Saint-Ange - commerciale - il s'agit d'un noud important du commerce des fourrures - et agricole - quelques colons s'installent, une vingtaine sont là en $1758^{19}$ qui cultivent du blé, du tabac, du maïs, dans un système qui rappelle surtout celui du Pays des Illinois voisins, c'est-à-dire avec une main-d'œuvre en partie constituée d'esclaves. Les agents du roi comme de l'évêque de Québec assurent les fonctions de contrôle colonial, dans le contexte qui n'en demeure pas

15. Le terme de «Français» sera adopté tout au long de cet article, plutôt que "Canadien» par exemple, car il s'agit du terme employé à l'époque, tant par les habitants de Vincennes que par les observateurs.

16. Cité dans Paul C. Phillips, "Vincennes in Its Relation to the French Colonial Policy", Indiana Magazine of History, XVII,4 (december 1921): 336.

17. Winstanley Briggs, op. cit.

18. Andrew Cayton, Indiana..., op. cit., 68.

19. Lettre du chevalier de Kerlerec, 12 décembre 1758, cité dans Paul C. Phillips, «Vincennes...», op. cit., 335. 
moins relativement souple qui caractérise les possessions françaises du Pays d'en Haut. Le maigre foyer de peuplement français ne peut tenir que par l'étroite collaboration avec les Piankashaws voisins comme avec les tribus plus lointaines, d'autant plus que le commerce requiert une bonne entente entre les parties, qui ne supprime pas les incompréhensions mais c'est sur celles-ci qu'une nouvelle société germe lentement.

L'année 1763 marque un tournant dans le sens où la double autorité française disparait. Or, les Britanniques ne se soucient guère de la remplacer, et laissent Vincennes hors de leur champ d'intervention puisque le village se situe au cœur des terres qui doivent demeurer indiennes après 1763, sans implantations coloniales. Le sentiment dominant dans les sphères autorisées est que ce type d'implantation, comme le signale Gage, est avant tout gênant pour l'ordre colonial nouveau.

Il faut donc d'abord reprendre ce maigre dossier documentaire de l'année 1772, lorsque Gage décide d'une évacuation forcée de Vincennes au nom d'une bonne gestion des territoires nouvellement britanniques, et que les habitants s'y opposent à coup de pétitions. En soi cette prise de parole locale est importante, elle dit que la passivité, que trop de commentateurs américains ont associée avec l'oisiveté aux colons français, n'a pas cours à Vincennes. C'est donc de l'argumentaire donné par les habitants de Vincennes le 18 septembre 1772 qu'il faut partir, avant d'éclairer la question par des sources religieuses moins connues ou utilisées trop pour elles-mêmes, sans volonté d'intégrer le religieux au récit global de l'histoire.

Ce 18 septembre 1772, les habitants signent une pétition et un mémoire - rédigé par Jean-Baptiste Racine, dit Sainte-Marie, le commandant du poste - destinés tous deux au général Gage. Ce mémoire veut contrer point par point les accusations de ce dernier. Position a priori délicate, car il s'agit de s'opposer à l'autorité royale, mais geste qui semble aller de soi à la fois parce que Vincennes est menacé de disparition et parce que la communauté se sent outragée par l’image que lui renvoie le général britannique. Il s'agit donc d'abord pour les signataires - qui ne sont pas l'ensemble des habitants - de se définir en tant que communauté, et dans leurs relations à la Couronne comme au contexte local. En usant d'un procédé habituel qui veut que l'on attaque non pas le roi mais ses mauvais conseillers, les habitants déclarent la proclamation de Gage nulle sur le fond:

Nous nous Sommes Meconnues dans les termes de la proclamation de Vostre Exelence et ces mesme termes Nous Rassurent Sur l'avenir puisqu'il paroist 
quon enveut qua des Vagabons, aulieu que nous formons un peuple D'habitent tranquil cultivent des terres que Nous avoit concédé Sa Mageste Tres Chretienne, ou que Nous avons acheté et Souvent arozé de Nostre Sang tous titres aussi Sacré quinviolables Sous un gouvernement dont le fondement innebrenlable est assis sur la justice et Lequité ${ }^{20}$.

L'argument de la communauté de vagabonds sans loi ne tient pas, en conséquence, d'après les Français eux-mêmes. Ils s'appuient au contraire sur le droit et un ancrage historique certain pour dire leur légitimité à demeurer sur place, quitte à faire jouer l'héritage français contre l'autorité britannique, en rappelant implicitement les engagements de la Couronne à respecter les habitants des terres conquises. L'essentiel demeure pourtant dans la réfutation du vagabondage: non, les Français ne correspondent pas à l'image convenue du coureur des bois gyrovague que veut répandre Gage, mais ont bien fondé une communauté paysanne stable. C'est au nom de la nature de l'établissement de Vincennes que les arguments suivants sont avancés.

Et ceux-ci sont complexes. Ils dessinent en fait les rapports que les habitants entretiennent avec les Britanniques comme avec les Indiens. Avec les deux l'ambivalence règne. Les Britanniques sont mis en accusation de manière virulente, en effet, mais non pas pour leur tyrannie, leurs abus de pouvoir. Les Français de Vincennes reprochent à l'autorité coloniale son extrême faiblesse et non sa tutelle envahissante. C'est que la présence britannique dans l'ancien Pays d'en haut est en effet des plus ténues, puisqu'il s'agit de faire de la région une grande réserve commerciale, donc indienne, et non pas de se lancer dans une entreprise de reprise en main telle qu'elle a lieu alors dans les 13 Colonies. Ainsi, «quant au reproches qu'on Nous a fait de Vivre Sens loix et Sens Gouvernement, C'est une perre qui les fait a des enfants incapables de Se conduire Euxmemes cest aluy dy pourvoir ${ }^{21} »$.

Le rapport de soumission est affirmé, en même temps que l'autorité critiquée dans sa manière de faire. De même si le commerce faiblit c'est parce que les cadres coloniaux font défaut: «qu'on nous envoye des trouppes on voira toutte chauze Rentrer dans l'ordre le commerce renaitre $^{22}$ », d'autant plus que les colons font valoir la valeur stratégique de Vincennes, entre Détroit, le fort Pitt et le Pays des Illinois. Il existe donc

20. Cité dans Florence Goold Watts, «Some Vincennes Documents of 1772 », Indiana Magazine of History, XXXIV,2 (June 1938): 208.

21. Ibid, 209.

22. Ibid. 
une demande de la part de Vincennes: bénéficier d’un retour du contrôle colonial dans la région, qui passerait sans doute, même si ce n'est pas explicité dans la pétition, par un renoncement aux principes de la Proclamation de 1763 mis en application par Gage, et donc par une réintégration de Vincennes au monde "civilisé».

Les Indiens, eux, sont au fil du mémoire des miroirs ambigus de la nature des habitants. On peut déceler dans les propos tenus à Vincennes la tentation d'assimilation de tous les peuples de la région de manière égalitaire: l'accusation de Gage que les Français ne sont que de méprisables chasseurs - or dans la culture du temps celui qui chasse pour vivre est ravalé au rang de sauvage ${ }^{23}$ - ne tient pas puisque ces mêmes Français disent l'habitude partagée par tous. Français, Anglais, Indiens, tous chassent, à double titre, pour vivre et pour faire du commerce. Aucune hiérarchie culturelle, donc, sous la plume du commandant Sainte-Marie, mais plus loin une nuance à apporter: "Quent aux Seauvages Nous Serions trop heureux Sils avoient pour nous les attentions que labandons ou Nous Sommes nous force d'avoir pour eux cedont nous ne pouvons Nous flatter quantent que Nous nous pretons Un mutuel Secours avec une garnison que Nous desirons inutillement depuis long temps ${ }^{24}$.»

C'est en fait le principe même de la colonisation de la région qui est ici en jeu, autour du «mutuel secours» que Français et Indiens se portent sans cesse. Force est de constater que la relation semble évidente, la porte n'est pas fermée au voisin piankashaw, loin de là. Mais la relation est très inégale, et dominée encore par les Indiens, qui imposent très visiblement les termes de l'échange dans un sens qui leur est favorable ${ }^{25}$. Or la situation n'a pas l'heur de plaire aux Français, qui une fois de plus déplorent leur abandon et réclament une garnison britannique afin de rééquilibrer le rapport de force local.

Ainsi le rapport de dépendance envers les Indiens, s'il est trop flagrant, est mal accueilli; on lui préférerait sans doute un soutien administratif et militaire qui permettrait de profiter des occasions commerciales offertes par la présence indienne sans subir le pouvoir des tribus. Si un terrain commun apparaît bien - faut-il encore le décrire comme un «middle

23. Daniel Justin Herman, Hunting and the American Imagination (Washington, Smithsonian Institution Press, 2002), $356 \mathrm{p}$.

24. Cité dans Florence Goold Watts, «Some Vincennes... », op. cit., 209.

25. Situation au demeurant classique en Amérique du Nord avant que le peuplement angloaméricain ne s'impose. Des exemples dans Kathleen DuVal, The Native Ground..., op. cit.; Pekka Hamalainen, The Comanche Empire (New Haven, Yale University Press, 2008), 512 p. 
ground»?-, il n'est pas forcément perçu comme un idéal, et chaque groupe tente de tirer profit d'une situation instable. Et les habitants de Vincennes sont dans ce cadre mécontents de leur sort. Ou du moins affirment-ils haut et fort que leur établissement serait viable si la Couronne britannique voulait bien marcher dans les pas de la France et entretenir effectivement une présence forte dans la région. L'attitude est donc ambivalente, car elle mêle une demande de contrôle accru qui viendrait de l'autorité coloniale, mais avant tout pour perpétuer la communauté telle qu'elle existe depuis près d'un demi-siècle, et non pour s'inscrire dans le projet global britannique. La tutelle britannique n'est en l'état acceptée, revendiquée, uniquement en tant qu'elle permet de résoudre les problèmes locaux.

L'autorité ecclésiastique ne donne pas plus satisfaction. L’année 1763 marque certes la fin du Régime français mais aussi l'expulsion des Jésuites. L'ordre en était arrivé dans la nuit du 23 au 24 septembre, et il ne souffrait pas de contestation. Les Kaskaskias comme les Français tentèrent en vain de protester. Seuls deux prêtres sont donc présents après cette date dans le Pays des Illinois et la région de Vincennes: Collet, un Récollet, occupe la cure de Fort de Chartres jusqu'à sa mort en 1775, et Meurin, un Jésuite qui obtient de revenir dans sa mission indienne et décède en 1777. Lorsqu'il avait quitté les Kaskaskias, il leur avait suggéré de s'organiser entre laïques afin d'assurer le mieux possible la continuité du culte ${ }^{26}$. Il a fait de même à Vincennes, en font foi deux témoignages. Un Récollet de Détroit, Bocquet, écrit à $\mathrm{M}^{\mathrm{gr}}$ Briand, évêque de Québec dont dépend encore la région, au sujet d'un problème qui agitera longtemps l’Église catholique dans les terres sans clergé: «l'on m’a déjà assuré que le seul missionnaire cy-devant de la compagnie de Jésus et maintenant séculier, qui dessert la partye du Mississippi restée aux françois et depuis cédée aux espagnols, avoit donné au Notair la puissance pour recevoir le consentement des parti et leur permettre l'habitation ${ }^{27}$ ».

Ce notaire, Philibert, confirme lui-même pour un autre sacrement: "voyla depuis le départ du révérend père cinquante enfants a qui jay donné leau du baptême toujours dans léglise, le père me layant ordonné pour assujetir nos habitans a toujours reconnoitre la ste église ${ }^{28} »$. De même Philibert, le dimanche, donne un sermon et, tous les jours, assure la prière.

26. Sur l'expulsion des Jésuites du Pays des Illinois, Cécile Vidal, Les établissements français..., op. cit., 105-110.

27. Archives de l'archevêché de Québec (AAQ), lettre de Bocquet à M ${ }^{\text {gr }}$ Briand, 30 juin 1767.

28. AAQ, lettre de Philibert à $\mathrm{M}^{\mathrm{gr}}$ Briand, 6 avril 1767. 
La solution de Meurin semble à première vue à la fois pragmatique et allant dans le sens de l'autonomie locale. On aurait alors une communauté française qui parviendrait à la gestion entre soi de la chose religieuse, sans rapport à la hiérarchie. Mais outre le fait qu'il s'agissait dans l'esprit du promoteur de la chose d'un pis-aller en attendant le retour des jours meilleurs, l'option des rites catholiques assurés par un laïque ne satisfait personne, ni l'Église ni Philibert.

En effet, Bocquet soumet le cas à son évêque parce qu'il lui semble évident que les mariages passés devant Philibert seul sont nuls, et qu'il faut donc que les présumés époux passent de toutes façons par l'église, même des années après leur "mariage». On est là devant un cas proche de ces mariages entre coureurs des bois et Indiennes, qui étaient acceptés par les communautés locales mais que les missionnaires de passage tentaient de régulariser. Il s'agit pour l'Église de ne pas se laisser déposséder d'un sacrement qui fonde l'ordre social. Philibert lui-même constate que cette pratique mène à l'éloignement de la religion, et que son rôle n'est pas satisfaisant. Dans l'Église catholique, en effet, le rite, qui est à la base du lien social et hiérarchique, ne peut être opéré que par un prêtre. Sans prêtre, c'est la société qui est menacée ${ }^{29}$. C'est pour cela que Philibert prend sa plume pour s'adresser lui-même à $\mathrm{M}^{\text {gr }}$ Briand le 6 avril 1767, en accompagnement d'une pétition de certains des Vincennois qui malheureusement a disparu des archives.

Deux ans plus tard, le 22 avril 1769, en l'absence de réponse de l'évêque, qui a envoyé un prêtre à Kaskaskia mais pas à Vincennes, une deuxième pétition est envoyée, rédigée par Sainte-Marie. Il faut d'abord s'interroger sur sa représentativité, afin de comparer cette prise de parole face à l'autorité religieuse avec celle qui suivra, en 1772, face à l'autorité politique. En 1772, 61 personnes signent (ou marquent d'une croix) le mémoire. Si l’on considère qu'en 1770 le père Gibault comptait 80 chefs de famille "qui sèment» et de nombreux habitants exerçant d'autres métiers ${ }^{30}$, et même en considérant qu'entre les deux dates la population a augmenté, c'est sans doute la majorité de la population stable de Vincennes qui s'implique, car la cause est majeure. En 1769, on ne comptait que 16 signatures, dont 6 en commun avec le texte de 1772 - on ne compte pas, par exemple, François Bosseron, peut-être le marchand le plus important de la région.

29. Ollivier Hubert, Sur la terre comme au ciel..., op. cit., 95-96.

30. AAQ, lettre de Joseph Gibault à $\mathrm{M}^{\mathrm{gr}}$ Briand, 1770. 
La volonté de retour à un strict contrôle religieux serait-elle l'affaire d'une faction minoritaire? Ces hommes craignent avant toute chose une rupture dans la chaîne des temps: les cinq années sans prêtre sont bien une anomalie, "qui eface les encienne principe des religions que nous avons reçu de nos encestre». Or cette rupture n'est pas seulement religieuse, car les signataires ont intégré pleinement le discours ecclésiastique, ils voient dans la fin de la présence du prêtre, plus encore sans doute que dans celle de l'autorité politique car celle-ci peut se reconfigurer à l'échelle locale, un risque pour l'ordre social: c'est la décomposition de la communauté traditionnelle qui est entrevue. Alors vient le temps des «enfants qui nont pas de crainte de leur propre père $[\ldots]$ et fuyant toujours le chemin de la vertu ». Pour retrouver ce chemin, il y a réaffirmation explicite de «la grande naicessité quil y a davoir un chef pour nous tenir tout dans la voix du salut».

Ce vide violemment perçu tient au fait que les rites qui ordonnent le monde, fondent le social, ne sont plus assurés. Sans sacrements, notamment, les vies se fragilisent. Du moins une minorité d'habitants le font savoir. Quant à connaitre le sentiment des fameux enfants qui refuseraient l'autorité paternelle, l'histoire sociale comme religieuse de Vincennes ou du Pays des Illinois en général, avant comme après 1763, demeure très difficile à atteindre.

Le père Gibault y aide quelque peu, bien que son témoignage complique encore l'interprétation. En effet, $\mathrm{M}^{\mathrm{gr}}$ Briand a bien envoyé un prêtre aux Illinois, le Canadien Pierre Gibault, personnage objet de moult polémiques $^{31}$. Mais un prêtre seul, et basé à Kaskaskia, car la fermeture temporaire du Séminaire de Québec à la suite de la Conquête a empêché l'envoi de jeunes missionnaires en des terres si lointaines. Gibault, ordonné le 19 mars 1768 après une formation rapide, quitte Montréal le $1^{\text {er }}$ juillet lesté d'instructions de $\mathrm{M}^{\mathrm{gr}}$ Briand qui tendent à appliquer au Pays des Illinois les règles canadiennes, prenant ainsi le contre-pied de la Couronne britannique: prêcher la "grande docilité» envers le nouveau pouvoir et reconstruire une société chrétienne ${ }^{32}$. Le grand âge de Meurin fait que c'est à Gibault qu'échoît la tâche de visiter les postes isolés. Il vient pour la première fois à Vincennes peut-être en mars 1770. Sa relation de l'événement, dans une

31. J. P. Dunn, "Father Gibault: The Patriot Priest of the Northwest», Transactions of the Illinois State Historical Society, 1905 ; Joseph P. Donnely, S.J., Pierre Gibault, Missionary, 1737-1802 (Chicago, Loyola University Press, 1971), 199 p.

32. Archives de l'Université Notre Dame, CAVI 1/09, instructions de $M^{\text {gr }}$ Briand au père Gibault, 1768 . 
lettre à son évêque, est ambivalente. D’un côté, il pointe «la religion presque éteinte», «le libertinage et l'imdévotion», en affirmant que "tout s'est relâché». Mais d'un autre côté, il livre ce récit de son arrivée:

A mon arrivée tout le monde est venu en foule me recevoir au bord de la Rivière ouabache; les uns se jettoient a genoux sans pouvoir parler, les autres ne parloient qu'en sanglotant, les uns crioient mon père sauvez nous, nous sommes presque dans l'enfer; les autres disoient Dieu ne nous a donc pas encore abandonné, car c'est lui qui vous envoie vers nous pour faire faire pénitence de nos péchés; les uns disoient, ah Mr que n’ètes vous pas venu il $\mathrm{y}$ a un mois, ma pauvre femme, mon cher père, ma chère mère, mon pauvre enfant ne seroit pas mort sans sacrement ${ }^{33}$.

Faisons ici la part de la perception du prêtre, missionnaire qui arrive pour la première fois dans un poste, comme de la nature performative de l'écrit en question: il s'agit d'éclairer l'évêque, d'insister sur le besoin en prêtres d'une population en attente. Il n'en demeure pas moins que l'insistance sur les sacrements montre bien qu'un manque est ressenti depuis 1763 : le rite n'est plus assuré, l'incertitude règne sur le sort des vivants et, plus encore, des morts.

Aussi le rôle de Gibault est-il d'assurer ces sacrements. Mais en 1770 il ne reste guère, et il ne reviendra, quelques jours, tout au plus quelques semaines, qu'en 1771, 1775 et 1777 avant que l'invasion américaine ne lui donne un autre rôle. Chaque fois, sa tournée missionnaire consiste en l'administration tardive de sacrements. Ce qui ne signifie pas qu'en son absence le rite s'éteigne: les registres de la paroisse Saint-François-Xavier signalent que Philibert continue son œuvre en baptisant et en enterrant, sans célébrer de mariages; Étienne Juteaux, «gardien de l'église», célèbre de même trois enterrements en avril $1770^{34}$. Mais sans prêtre, le rite demeure inopérant, et la présence discrète d'un capucin, Chapert, qui signe neuf actes en 1771, ne peut faire oublier l'essentiel, à savoir que le maintien de la foi comme de l'ordre ne peut, pour certains au moins, être pensé sans présence de clergé.

Cependant les registres de la paroisse révèlent autre chose. Largement sous-exploités par les historiens, ils révèlent, outre la présence permanente de quelques esclaves d'origine africaine, six mariages célébrés par Gibault en 1775 et 1777 . Sur les 12 conjoints, la moitié sont des Vincennois de

33. AAQ, lettre de Gibault à $\mathrm{M}^{\mathrm{gr}}$ Briand, mars 1770.

34. Archives de l'Université Notre Dame, St. Francis Xavier Church, Vincennes, Parish Records, type copy, M93\#23. 
naissance. Les autres sont originaires de Ouatanon, La Mobile, Roc - au Québec -, le Détroit, la Prairie de la Magdeleine et Montréal. Par ailleurs on ne remarque aucune épouse indienne: le rapport quotidien, commercial, diplomatique, religieux peut-être, ne signifie pas - plus? - qu'une société métisse se constitue ${ }^{35}$.

Il faut ajouter à cela qu'alors que Gibault évalue en 1770 les habitants à 80 agriculteurs pour 700 à 800 personnes au total, la liste, établie par le Land Office américain, des propriétaires à la date de 1783 donne 152 noms, dont 149 d'origine française (les trois autres sont Frederick Mehl - de Philadelphie -, Francis Vigo - un ancien soldat italien des armées espagnoles - et Ann, veuve de Moses Henry; les deux premiers se sont intégrés à la communauté française $)^{36}$. Le doublement de la population française est improbable - le colonel Harmar compte, lui, 900 habitants français et 400 américains en $1787^{37}$, mais tous les chiffres pour la période sont à manier avec une extrême précaution -, pour autant il semble donc, d'une part, que Vincennes soit demeuré une communauté très dynamique d'un point de vue démographique durant la période britannique au moins, et d'autre part que ce dynamisme soit aussi celui d'une Amérique française qui continue de courir du golfe du Mexique à l'embouchure du SaintLaurent jusqu'au XIx ${ }^{\mathrm{e}}$ siècle. Il faut donc inscrire Vincennes dans la croissance de celle-ci, qui se poursuit en fait après 1763, après la disparition de la tutelle politique française ${ }^{38}$.

Peut-on à l'aune de ces quelques indications repenser le passage en 1778-1779 à la tutelle américaine? Lorsqu'en 1778 George Rogers Clark et ses hommes, stationnés à Kaskaskia qu'ils viennent de conquérir au nom des Insurgents américains, veulent prendre Vincennes, ils choisissent d'abord de tenter de convaincre les habitants de changer de souveraineté. Pour ce faire, Clark envoie sur place un marchand, Jean-Baptiste Lafont, et le père Gibault. Leur mission est une réussite, Vincennes accueille Clark sans opposition et prête serment aux nouvelles autorités. Gibault aura à justifier de son rôle auprès de son évêque, puisque selon toutes les appa-

35. Kathleen DuVal, «Indian Intermarriage and Métissage in Colonial Louisiana», William and Mary Quarterly, LXV,2 (April 2008): 267-304.

36. History of Knox and Davies Counties, Indiana, From the Earliest Times to the Present (Chicago, The Goodspeed Publishing Co., 1889), 110-112.

37. Andrew Cayton, Frontier Indiana..., op. cit., 112.

38. Jay Gitlin, «On the Boundaries of Empire: Connecting the West to Its Imperial Past», dans William Cronon, George Miles et Jay Gitlin, dir., Under An Open Sky: Rethinking America's Western Past (New York, Norton, 1992), 82, et Negotiating the Course of Empire: The French Bourgeois Frontier and the Emergence of Mid-America, 1763-1863, thèse de doctorat, Yale University, 2002. 
rences il aurait donc participé à la subversion de l'autorité politique. Mais le témoignage de Lafont est de ce point de vue éclairant: «j’en ay eu seul la direction [de la mission politique] luy meme s'en etant tenu envers les uns et les autres a la seulle exhortation tendante a la paix et a l'union et a empecher l'effusion du sang ${ }^{39}$ ".

L'attitude des Français peut-elle dès lors s'expliquer par l'acquiescement local de l'autorité de contrôle religieux au changement d'autorité politique? Au nom justement de l'obéissance due au pouvoir civil que rappelait $\mathrm{M}^{\mathrm{gr}}$ Briand dans ses instructions, Gibault aurait convaincu les Français de s'adapter aux Américains, qui paraissaient devenir les nouveaux mâ̂tres. Gibault et Briand auraient juste eu une vision différente du détenteur du pouvoir local: Briand s'arc-boutait sur l'autorité britannique comme substitut encore acceptable à celle du roi de France, mais localement Gibault reconnaissait le fait de guerre, la victoire américaine. Mais si Gibault n'avait pas toute l'influence désirée, si Vincennes n'était pas unanime dans son rapport à l'Église?

C'est alors directement le rapport au politique qui joue : les Britanniques avaient menacé Vincennes de disparition, avaient refusé de jouer leur rôle de protecteurs, pourquoi ne pas essayer une nouvelle solution pour, toujours, assurer la pérennité et la prospérité locale? François Bosseron, qui n'avait pas signé l'appel à $\mathrm{M}^{\mathrm{gr}}$ Briand mais avait participé à celui à Gage, vendait des vivres aux Britanniques avant 1778, aux armées américaines lorsque celles-ci sont arrivées, et de nouveau aux armées britanniques lorsqu'elles ont brièvement repris Vincennes en décembre de la même année ${ }^{40}$. L'essentiel est bien la poursuite d'objectifs locaux, voire individuels, et donc les luttes géopolitiques à l'échelle continentale.

La prise de parole collective, l'affirmation d'une gestion locale comme la possibilité d'un détachement des structures ecclésiastiques pourraient laisser penser à un chemin vers une forme de modernité, mais dans le même temps la présentation de requêtes aux autorités de la part d'une communauté locale, d'un corps en quelque sorte, comme la demande d'une partie au moins des habitants d'un retour au cadre paroissial peuvent être assimilées à une volonté de continuité des structures d'Ancien Régime. Que change alors le nouveau transfert de souveraineté, puis la modification de la tutelle ecclésiastique?

39. Lafont à Clark, 7 août 1778, cité dans «Father Pierre Gibault and the Submission of Post Vincennes, 1778", American Historical Review (1909): 551.

40. Janet Shaw, dir., "Account Book of Francis Bosseron", Indiana Magazine of History, 25,3 (September 1929): 212-241. 


\section{ENTRE DEUX OBÉDIENCES, 1778-1795}

En effet, 1778 ne constitue sans doute pas, à l'échelle locale, le tournant attendu. Et ce parce que la conquête est en fait largement inachevée. La Guerre d'Indépendance ne prend fin qu'en 1783, et le traité de Paris ne signifie rien de précis quant à une fin des hostilités entre les Appalaches et le Mississippi. D’abord parce que les Britanniques, dans les faits, ne lâchent guère prise et s'accrochent à leur pouvoir dans la région, ensuite, et surtout, parce que les tribus indiennes qui avaient soutenu la cause britannique ne cessent pas le combat, assurées qu’elles sont qu’une défaite finale signifierait la fin de leur présence sur les territoires en jeu. La région de Vincennes est donc encore longtemps au cœur des conflits, lors d'une étape cruciale de la conquête américaine du continent ${ }^{41}$.

Pendant une dizaine d'années, il n'y a en fait pas d'installation réelle de structures administratives américaines à Vincennes. Seule existe une cour de justice créée par l'État de Virginie et dont l'autorité face aux squatters qui investissent sans cadre légal les environs de Vincennes est très réduite. Reste que le corps "civique» du Vincennes français et catholique survit, lui, même si les traces en sont une nouvelle fois ténues. Les registres de la paroisse montrent encore à la fois l'absence de clergé régulier jusqu’à ce que Gibault revienne de 1784 à 1789, et en même temps le maintien des rites, des rythmes et des structures. C'est toujours Philibert qui officie, avec parfois le passage de Payet, un prêtre inconnu, et de Gibault. L'essentiel réside dans la permanence du rythme des baptêmes, des mariages, des sépultures chrétiennes et des marqueurs sociaux.

La société de Vincennes demeure socialement hiérarchisée. Les registres différencient bien les habitants des "Sieurs» et des "Dames», les notables. Parmi ceux-ci, bien sûr, les descendants des seigneurs du lieu: sieur Antoine Drouet de Richerville épouse Marie Vaudrie le 18 juillet 1779 , sieur Jean-Baptiste Legras, colonel de la milice du commandant du fort, épouse quatre jours auparavant dame Marie Desvegnets. Mieux encore, le 6 juin 1785, Gibault marie le sieur Pierre Gamelin, marchand et interprète, avec dame Marguerite Drouet de Richerville; les témoins sont au nombre de quinze, parmi lesquels Legras, François Bosseron,

41. Pour une vision synthétique, voir Colin G. Calloway, One Vast Winter Count: The American West Before Lewis and Clark (Lincoln, University of Nebraska Press, 2003), 313-366, qui offre une intégration de la révolution américaine à l'histoire de l'Ouest, et Gary B. Nash, The Unknown American Revolution. The Unruly Birtb of Democracy and the Struggle to Create America (New York, Penguin, 2005), 512 p., pour l'intégration inverse des «guerres indiennes » à l'histoire de la révolution américaine. 
Francis Vigo et jusque Pierre Chouteau venu de St. Louis participer aux réjouissances, signe que Vincennes fait bien encore partie d'un univers commercial qui préexistait aux Américains ${ }^{42}$.

Les quelques comptes des marchands locaux qui subsistent pour ces années le révèlent assez: les affaires sont ralenties par la guerre parfois, mais elles se poursuivent pour les Gamelin, Bosseron, Vigo et les autres, qui lient toujours par leurs réseaux commerciaux Vincennes à Détroit et à St. Louis ${ }^{43}$. Voire à des destinations plus lointaines: le 3 décembre 1785, Gibault bénit la cloche de son église, que François Bosseron a fait acheter à Philadelphie ${ }^{44}$. Ce qui révèle à la fois que Vincennes n'est pas un poste isolé et qu'au moins certains de ses notables prennent soin de participer à la vie de la paroisse, à faire en sorte que le cadre catholique soit maintenu.

Le témoignage qu'en a laissé Gibault lui-même tendrait à aller dans le même sens. Nous ne disposons que d'une lettre pour cette période, datée du 6 juin 1786, à un moment très pénible des relations entre Français, Américains et Indiens, situation à laquelle le prêtre n'attache que très peu d'importance. Face à l'évêque de Québec, dont il dépend toujours même s'il avoue lui-même que les choses ne lui sont plus très claires depuis la nomination en 1784 de John Carroll comme préfet apostolique pour les États-Unis, il décrit avec précision sa paroisse de Vincennes. Son laïus est d'abord positif. Le retour d'un prêtre stable s'est fait sans heurts, même avec succès :

Il y a un an et demi que je suis ici, et quand j'y suis arrivé je n'ay trouvé personne ny grand ny petit pour servir la messe qu'un vieil européen qui ne pouvoit pas toujours venir et alors point de messe. Deux mois après j'en avois plusieurs, et à présent jusqu'aux plus petits du village non seulement savent servir la messe mais les cérémonies des festes et dimans et tout le catéchisme petit et grand.

Dans le même temps, les notables - «quelques uns des principaux marchands et habitants» - font de remarquables efforts - «tout ce qui dépend d'eux» - pour maintenir ordre et autorité, sous le commandement de Legras et en l'absence de structure officielle supérieure à l'échelon local. Pour finir, Gibault a obtenu, non sans devoir laisser craindre qu'il

42. Archives de l'Université Notre Dame, St. Francis Xavier Church, Vincennes, Parish Records, type copy, M93\#23.

43. Christopher Coleman, «Letters from Eighteenth Century Indiana Merchants », The Indiana Magazine of History, V,4 (December 1909): 137-159.

44. Archives de l'Université Notre Dame, St. Francis Xavier Church, Vincennes, Parish Records, type copy, M93\#23. 
puisse abandonner la paroisse, qu'on lui bâtisse une église, encore inachevée au mitan de 1786, mais dont la cloche, on le sait, est arrivée six mois auparavant. Tout va bien, donc.

Et pourtant, plus loin dans sa missive, Gibault dresse un tableau très noir des mœurs et de la moralité locales: «ici tout est barbare». Libertinage et ivrognerie semblent régner, menant droit au crime, à la dissolution des mours: «les ménages brouillés, les pères et mères en discorde avec leurs enfants, des filles subornées et enlevées dans les bois, mille autres désordres que vous pouvez inférer de ceux $\mathrm{cy}^{45}$ ». Que dire alors? Il semblerait que Vincennes se ressemble, en fait, et qu'effectivement le passage très formel encore sous administration américaine ne modifie pas l'établissement qui dans les années 1760, demandait, et obtenait l'assistance de son évêque: un même attachement au cadre, aux rites, à la présence d'un prêtre garant des rythmes de vie, des sacrements; mais en parallèle, un respect des enseignements de la religion qui laisserait à désirer, et une forme d'ordre locale, en somme.

Néanmoins, dans ces mêmes années, cette dernière est mise en danger par l'afflux des colons américains, qui bouleverse à tous points de vue les équilibres locaux et régionaux. Le passage de George Rogers Clark aura au moins eu une conséquence majeure, l'arrivée de migrants anglo-américains, pour l'essentiel virginiens et pennsylvaniens. Ceux-ci présentent un profil différent des Français de Vincennes. Ils sont avant tout avides de terres, et donc de sa conquête de sa mise en valeur agricole à leur idée, et non de commerce avec les Indiens. Au contraire, leur caractéristique fondamentale est bien de voir ceux qui les précédaient comme des obstacles à leur propre mode de développement, fondé sur la volonté d'indépendance, de propriété, de conviction de leur supériorité, sur l'idée d'«improvement» qui suppose que nul n'a su tirer profit de la région avant eux et que nul comme eux ne sait faire avancer la «civilisation» dans les contrées sauvages ${ }^{46}$.

Leur pénétration de la région de Vincennes et leur installation peuvent être légales ou illégales. Dans ce dernier cas, il leur faut alors pétitionner,

45. Lettre de Gibault à $\mathrm{M}^{\mathrm{gr}}$ Briand, 6 juin 1786, dans «Father Pierre Gibault... », op. cit., 552-556.

46. Malcolm J. Rohrbough, The Trans-Appalachian Frontier: People, Societies and Institutions, 1755-1850 (New York, Oxford University Press, 1978), 444 p.; Eric Hinderaker, Elusive Empires: Constructing Colonialism in the Ohio Valley, 1673-1800 (New York, Cambridge University Press, 1997), 299 p. ; Patrick Griffin, American Leviathan : Empire, Nation, and Revolutionary Frontier (New York, Hill and Wang, 2007), 368 p. 
transiger avec un pouvoir d'origine à la fois américaine et française, car la continuité l'emporte et c'est bien Legras qui dirige à Vincennes la cour civile et criminelle qui émane de l'État de Virginie, qui à défaut d'une intervention supérieure, a pris en charge - ou plus exactement revendiqué la prise en charge, tant la tutelle est lointaine - l'administration du territoire $^{47}$ : leurs demandes sont en langue anglaise, bien sûr, rédigées comme elles le seraient ailleurs dans les nouvelles terres conquises ${ }^{48}$, mais la réponse peut être en français et porter la trace d'un autre monde, sous la plume de Legras: «Vu la présente requeste à moy presentée par les Srs ci-dessus denommé il est ordonné [par] le grand greffier de la cour de conceder et acorder a chaquun quatre cent arpents de terre pour létablissement du pais ${ }^{49}$.»

Deux cultures peuvent encore se rencontrer à Vincennes, et des AngloAméricains - à qui il est encore répondu en arpents - peuvent s'adresser avec déférence à un porteur d'autorité issu d'un autre monde que le leur, puisque celui-ci s'est vu accorder une parcelle de pouvoir par le vainqueur. Un notable français peut servir de relais local, au moins temporairement et faute de mieux, au pouvoir virginien. Pourtant ils sont minoritaires ceux qui, parmi les nouveaux colons, en passent par là, et ceux-là même qui acceptent d'emprunter la voie d'un strict et prudent légalisme ne disent rien de l'estime qu'ils portent à celui auquel ils s'adressent comme à ce qu'il représente culturellement.

Le fait est que sur le terrain, l'apparition de ces nouveaux colons est avant tout déstabilisatrice. Elle remet en cause tant la relation coloniale ancienne entre Français et Indiens, si imparfaite qu'elle fût, que les structures internes que les habitants de Vincennes s'évertuent par ailleurs à maintenir, à faire vivre. Deux problèmes sont préoccupants: le statut des terres des Français et la relation aux Indiens. Dans le premier cas, les colons anglo-américains qui arrivent avant la reprise en main fédérale de 1787 ont beau jeu d'affirmer qu'ils ne voient pas d'où les Français tiennent leur droit à la terre, ce qui leur permet de s'installer eux-mêmes en conquérants, considérant parfois les possessions françaises à l'égal des revendications indiennes, comme nulles.

Dans le deuxième cas, le problème réside dans les conceptions fondamentalement différentes que les Français et les Anglo-Américains se font

47. History of Knox..., op. cit., 96.

48. Indiana Historical Society, Albert Gallatin Porter Papers, Box 2, Folder 21, pétitions des 29 juillet et 4 septembre 1785 .

49. Ibid., réponse de Legras du 5 septembre 1785 . 
de leur place dans le contexte régional, les premiers ayant un monde à conserver et à étendre, les seconds un nouveau à créer sur une table rase, «to promote our hapiness» comme l'affirment les 71 signataires d'une pétition datée du $1^{\mathrm{er}}$ juin $1786^{50}$ en écho à la "pursuit of happiness » de la Déclaration d'indépendance des États-Unis.

Le 22 du même mois, John Small, un colon, rapporte à George Rogers Clark une anecdote éclairante. Dans le cycle de violence qui a comme origine l'installation des colons et le droit qu'ils s'arrogent de voler les terres comme de tuer les Indiens, un événement somme toute banal qui pour finir voit un parti de colons s'emparer d'un Indien - un Piankashaw selon toute vraisemblance - pour l'assassiner entraine une conséquence inattendue pour les Small, Donnally ou Sullivan engagés dans l'affaire:

Next Day we weare called together by Co. Legrass Who Was Very much Exasperated Against Us for the putting of the savage to Death in consequence of which the Heads of the French Inhabitants Gave us a piece ow writeing ordering Every American that can not produce a passport to leave this place Bagg and Baggage Immediately. Mr Sullivan produced a pass but they would not Look at it nor Give him no heering but ordered him to leave this place with his family at his own Risk and Peril without Delay the reason why he Received the above mentioned orders was in Consequence of putting the affore mentioned Indian. [...] [another] Day the French ascorted several Indians into the Town which Indians are shuer are our Enemys the French has repeatedly told us that they would keep the Indians at this place ${ }^{51}$.

Tel qu'ils sont décrits par un Anglo-Américain, les camps semblent clairs: en cas de conflit, les Français choisissent d'expulser les colons plutôt que les Indiens. Or ce sont bien les Français qui localement sont détenteurs de l'autorité. En conséquence, le 28 juin, Daniel Sullivan lui-même prend la plume pour défendre son cas devant Clark et en appeler à des renforts armés pour défendre les positions des colons anglo-américains ${ }^{52}$. Le mois suivant, Legras envoie Bosseron, accompagné d'Antoine, Paul et Pierre Gamelin et de Lachine, en mission de conciliation chez les Piankashaws. Ces derniers répondent qu'ils n'en ont qu'après les Américains, que les Français n'ont eux rien à craindre, et alors que la paix semble possible malgré tout, la nouvelle se répand qu'un fort parti angloaméricain a attaqué des Piankashaws du groupe disposé à la paix et un

50. Pétition reproduite dans Leonard C. Hedelman, «Danger on the Wabash. Vincennes Letters of 1786 ", Indiana Magazine of History, XXXIV,4 (December 1938): 457-458.

51. Lettre de John Small à George Rogers Clark, 23 juin 1786, dans ibid., 460.

52. Lettre de Daniel Sullivan à George Rogers Clark, 28 juin 1786, dans ibid., 461. 
village des Miamis, sans s'enquérir de la nature d'alliés ou d'ennemis des Indiens en question. Les six morts et les sept blessés risquent de remettre une fois de plus en question la stabilité régionale. D’où la plainte répétée de Legras contre non pas l'ensemble des colons mais seulement les squatters illégaux, incontrôlables car non enregistrés auprès de la cour de Vincennes ${ }^{53}$.

Il y a donc bien, sur le terrain, opposition entre les deux groupes issus de deux colonisations différentes. Pour l'heure, les Français détiennent encore une part du pouvoir: ils sont plus nombreux, Legras est malgré tout dépositaire de l'autorité, et leur gestion du monde indien s'avère indispensable à la survie des colons anglo-américains. Mais il suffit que les équilibres évoluent, que l'autorité et le poids démographique échappent aux Français pour qu'ils s'avèrent inutiles, encombrants, que la tentation américaine de supprimer ceux qui préexistent l'emporte sur toute autre considération. Maintenir le statu quo peut présenter un intérêt temporaire, mais vient un moment où les Américains tiennent plutôt à imposer leurs solutions, leurs modèles.

L'année 1787 marque de ce point de vue un tournant dans l'américanisation juridique de Vincennes: d'une part, l'Ordonnance du Nord-Ouest offre un cadre administratif stable à la région et permet un encadrement plus strict, une prise en main par les Anglo-Américains des leviers du pouvoir; d'autre part, c'est John Carroll, depuis Baltimore, et non plus l'évêque de Québec, qui prend en charge les destinées catholiques de la région entre Appalaches et Mississippi.

Les résultats sont décalés, d'abord politiques avant d'être religieux. Car $\mathrm{M}^{\mathrm{gr}}$ Carroll, dans un premier temps, échoue dans son entreprise, faute de moyens adéquats. Pierre Huet de la Valinière, né dans le diocèse de Nantes, devenu Sulpicien à Montréal mais sorti de la congrégation, revenu en France et reparti en Amérique du Nord ${ }^{54}$, était sans doute un mauvais choix comme vicaire général au Pays des Illinois. Mais Carroll manquait de personnel, et ce prêtre en constante rupture n’a rien pu établir de fixe sur ses terres de mission, où il opère de 1787 à 1789 . Quant à Gibault, il quitte Vincennes après le 11 octobre 1789 , date de son dernier acte dans le registre paroissial, pour s'établir dans la Louisiane espagnole. De nou-

53. Lettre de Jean-Baptiste Legras à George Rogers Clark, 22 juillet 1786, dans ibid., 462467.

54. Lucien Lemieux, «Pierre Huet de la Valinière», Dictionnaire biographique du Canada en ligne, http :/ / www.biographi.ca /009004-119.01-f.php ?\&id_nbr=2467\&\&PHPSESSID=pso3lve50p169 h6qn7r917tfl1 
veau, Vincennes est une paroisse sans prêtre: Antoine Gamelin assure quatre baptêmes en décembre 1789, Pierre Mallet lui succède jusqu'en décembre 1792.

Les États-Unis sont parallèlement plus efficaces en termes d'implantation administrative que l'Église catholique. Le gouvernement américain, par l'Ordonnance du Nord-Ouest, "approchait le développement de l'Ouest avec un détachement émotionnel et un sens de son propre pouvoir proches du fantastique ${ }^{55}$ ", qui impliquait en tout cas qu'Indiens et Français, même si ces derniers se voyaient reconnaître quelques spécificités, se moulent, s'adaptent aux impératifs nouveaux, au modèle social et culturel proposé. C'est le lieutenant-colonel Josiah Harmar qui, à l'été 1787, est chargé d'annoncer le changement à Vincennes, mais il en part le $1^{\text {er }}$ octobre en y laissant le major John Francis Hamtramck, né à Québec et possédant une ascendance canadienne et catholique, ce qui le rendait idéal pour le rôle - et de fait il s'est intégré à Vincennes: dès octobre 1788, il était témoin d'un baptême, et à l'été 1790, il épousait Marie Perrot, dont il eut trois filles.

Deux problèmes se posaient: la structure du pouvoir et le partage des terres. Des manifestations d'insatisfaction, de divisions, tout comme le décès de Legras en février 1788, ont conduit Hamtramck à dissoudre la cour en avril et à organiser de nouvelles élections, dont les vainqueurs furent Jean-Baptiste Millet, Nicolas Baillarjon, Moses Henry, Thomas Dalton et James Johnson. Un premier tournant avait eu lieu, les Virginiens accédaient au pouvoir face aux notables canadiens. En 1790, de même, le pouvoir devait être partagé. Cette année-là, le gouverneur du Territoire du Nord-Ouest, Arthur St. Clair, laisse son secrétaire Winthrop Sargent se charger de la gestion locale. En juin, ce dernier organise le comté de Knox autour de Vincennes et nomme aux postes de responsabilité les Gamelin, Bosseron, Richerville, Vigo, mais aussi John Small - celui qui avait eu maille à partir avec Legras - James Johnson ou Luke Decker ${ }^{56}$.

Et c'est ce même Sargent qui est à l'origine du règlement de la question des propriétés, dont les titres étaient l'objet de tous les litiges. Derrière le problème juridique, c'était la conception de l'établissement des colons qui était en jeu. Ainsi, le 18 mai 1789, 20 Français signent une pétition enregistrée par Antoine Gamelin, "notaire du greffier». Et parmi les signataires, Nicolas Baillarjon, membre de la cour - qui par ailleurs fait partie

55. Andrew R. L. Cayton, Frontier Indiana..., op. cit., 106.

56. Ibid., 115-123. 
des 16 hommes sur les 20 qui ne font qu'apposer leur marque, sans savoir signer:

Nous habitants du Poste Vincennes soussignés et sous nos marques ordinaires, après avoir examiné la conduite de plusieurs des notres qui au préjudice du bien Et service public, s'approprient injustement des quantités d'emplacement dans la Commune, non pour y former des établissements suivant le principe des concessions, mais seulement pour cultiver Et y semer des grains, et plusieurs même en Font un trafic, et Les vendent impunement a des jeunes gens volontaires et qui ne sont point dans le cas de les Etablir avons êtes dopinions et Sommes tous Dopinions - Vu le tort que nous cause pareille conduite, qu'il soit expressement deffendu a toutes personnes quelconques d'occuper ny Cultiver aucune place ny piece de terre dans notre commune Jusqu'a ce qu'il en soit ordonné par le gouvernement ou autres a ce authorisé et approuvé, seulement qu'il soit permis a chaque famille de prendre et occuper un emplacement de vingt cinq toises sur toutes faces, pour y former un Etablissement Et y Batir Et non pas seulement pour cultiver comme plusieurs le prétende. Car ainsy fut dit et conclus en Lassemblée public tenue le dimanche 18 May $1789^{57}$.

Il s'agit là d'une situation complexe : qui sont «les nôtres» dont parlent les signataires? Faut-il comprendre que des Français adoptent un comportement accapareur et spéculateur? Que donc la communauté demeure divisée, certains se coulant dans les habitudes des nouveaux venus, tandis que d'autres arguent encore que l'idéal réside dans le maintien de l'ancienne coutume? Ce qui est certain c'est qu'il y a ici, de la part d'une partie de la communauté française uniquement - aucun Virginien ne signe - , une critique de l'accumulation spéculative des terres dans le contexte de la colonisation anglo-américaine. Et, somme toute, ce que ces Français défendent n'est pas loin d'un idéal jeffersonien demeuré toujours théorique, celui d'un monde de fermiers propriétaires de leurs terres et y faisant vivre leur famille, modèle qui est source de toutes les vertus républicaines. Pour ce faire, il faut faire appel à l'autorité, même si celle-ci demeure instable, que l'on ne sait pas encore réellement à qui il vaut mieux s'adresser, en quel pouvoir il vaut mieux espérer. Le tout est qu'un pouvoir garantisse les structures sociales, donc économiques et culturelles.

Ce que fait Winthrop Sargent s'apparente à cela. L'année 1790 est consacrée au recensement des titres d'avant 1783, et à l'établissement clair des propriétés. Les Français se voient garantir la possession des leurs, les colons anglo-américains la possession de lots égaux. Mais le processus n’est

57. Indiana Historical Society, Albert Gallatin Porter Papers, Box 2, Folder 21, pétition des habitants de Vincennes du 18 mai 1789. 
que mis en branle, il ne s'achèvera que des années plus tard, ne serait-ce que parce que Sargent, une fois de plus, n'est pas dépositaire officiel du pouvoir, il n'est que délégué par St. Clair faute de mieux - et en novembre 1794, une lettre de Pierre Gamelin, Louis Eudeline, Francis Vigo et James Johnson signalera encore à quel point Sargent comme St. Clair sont absents de Vincennes ${ }^{58}$. Dans l'histoire, la propriété française y aura beaucoup perdu, les descendants des premiers Vincennois seront dépossédés lentement, sans qu'il y ait besoin de mesures de confiscation ${ }^{59}$.

La situation politique est donc ambiguë, puisque des Français participent aux nouvelles structures du pouvoir, mais dans le même temps y sont mis en minorité, puisque les États-Unis garantissent les titres de propriété mais imposent un modèle qui brutalise les structures locales. L'américanisation du territoire est une œuvre douloureuse. Le paradoxe est que l'américanisation de la gestion de la paroisse est fondée à l'inverse sur l'idée d'un retour à un ancien régime plutôt qu'à la création d'une société neuve. Mais ce retour à l'ordre catholique n'est pas nécessairement mieux accepté que le bouleversement politique.

John Carroll, après l'échec de Huet de la Valinière, devait s'orienter vers d'autres solutions. Son séjour anglais de 1790 devait les lui apporter: Jacques-André Emery, supérieur du Séminaire de Saint-Sulpice, à Paris, a saisi l'occasion pour proposer ses services. Il s'agissait de permettre un développement missionnaire sulpicien tout en offrant une porte de sortie à un clergé refusant la Révolution française. Les religieux français devaient ouvrir et financer un séminaire, celui de St. Mary à Baltimore, mais aussi renforcer les rangs du clergé diocésain, le nouveau diocèse de Baltimore s'étendant jusqu'au Mississippi. Aussi le 8 avril 1791, quatre prêtres et cinq séminaristes quittent Saint-Malo. À la fin de l'année, trois prêtres et deux séminaristes les rejoignaient ${ }^{60}$.

Michel Levadoux, issu de la première fournée, est fait vicaire général pour le Pays des Illinois - Vincennes est inclus dans son ressort -, où il

58. Indiana Historical Society, Albert Gallatin Porter Papers, Box 2, Folder 24, letter à Winthrop Sargent, 4 novembre 1794.

59. Il convient de noter à ce propos l'insigne faiblesse de la littérature sur cette question des terres françaises et de leur sort sous le régime américain, à Vincennes comme ailleurs, comparativement à la richesse de l'historiographie sur les terres anciennement mexicaines dans le SudOuest. Une comparaison entre les deux situations, celle des Français du Pays des Illinois ou du Pays d'en Haut et celle des Mexicains s'avérerait pourtant d'une grande richesse quant à la compréhension du phénomène de la conquête par les Anglo-Américains de leur espace national.

60. Christopher J. Kauffman, Tradition and Transformation in Catholic Culture: The Priests of Saint Sulpice in the United States From 1791 to the Present (New York, Macmillan, 1988), chapitre 1. 
parvient en 1792. L'Auvergnat Benoît Joseph Flaget, de la deuxième fournée, futur premier évêque de Bardstown dans le Kentucky, est lui affecté à Vincennes, où son premier acte est daté du 29 décembre 1792. Flaget demeure en poste jusqu'en avril 1795 avant d'être rappelé sur la côte Est, et Levadoux jusqu'à 1796 avant de prendre poste à Détroit. Les deux hommes ont laissé peu de traces archivistiques de leur passage.

C’est le successeur de Flaget, Jean-François Rivet, qui éclaire quelque peu son œuvre. Flaget aurait bien commencé une certaine remise en ordre de la paroisse, aurait fait l'acquisition de «quelques propriétés» dans le village, aurait réussi son insertion dans le tissu local si l'on en juge par le fait qu'à deux reprises dans les années suivantes les habitants demandent à Carroll son retour ${ }^{61}$. C'est que Flaget était perçu comme un défenseur de la communauté, son porte-parole auprès de l'administration américaine. En témoigne une longue lettre que lui a adressée le 18 août 1794 le juge fédéral du Territoire du Nord-Ouest, George Turner. Turner accuse réception d'une nouvelle pétition des habitants de Vincennes, dont on n'a pas trace par ailleurs mais qui a été présentée par l'intermédiaire du prêtre. La réponse du juge est éclairante, même si l'on ne sait guère avec précision à quoi il répond, car il donne une leçon aux habitants de Vincennes:

The writer of the petition [...] contrasts (to little purpose indeed) the now situation of the people here, with that which they enjoyed under the French and British Governments. "Then», says he, "we were truly free. Every one could trade according to his means». To this I would answer, it is very possible the citizens might in those times, have been permitted to trade as they pleased. But surely this was no evidence of their being «truly free». We know, on the contrary, that at both those periods, the government was military - and consequently, despotic - In a legal sense, the inhabitants were slaves. Any privilege they enjoyed was a mere indulgence emanating from the commanding officer, and revocable at his pleasure or caprice. If this was freedom, it was a terrible - a detestable state of it [...].

Pray inform the people, good sir, that their present political situation rests on the secure basis of a government of laws - not of men; and that neither life, liberty nor property can be taken away from event the poorest and most friendless individual, without the concurrence of laws [...].

You are a man of science and respectability. [...]. Impress it, Sir, upon the members of your congregation, how indispensibly requisite it is to cultivate

61. Archives de l'Université Notre Dame, lettres de Jean-François Rivet à $\mathrm{M}^{\mathrm{gr}}$ Carroll, 6 août et 26 octobre 1796. Archives du diocèse de Covington, lettres de Jean-François Rivet à $\mathrm{M}^{\mathrm{gr}}$ Carroll, 4 mai 1797, 8 octobre 1798, 15 juillet 1799 . 
a general knowledge of the law. [...]. Advise them to reflect on the value of literary acquirements, that the rising generation may not live unenlightened for the want of a little education. Tell them that a people professing knowledge and morals can never be enslaved and hat knowledge once acquired can never be lost $[\ldots]$ that therefore knowledge is he treasure most to be prized ${ }^{62}$.

Il est difficile d'imaginer plus claire expression de l'incompréhension entre les deux modèles qui s'opposent alors à Vincennes: celui des habitants d'origine française qui demandent le respect des choses telles qu'elles furent parce que le contexte - les politiques impériales comme leur peu de poids souvent - leur permettait une liberté locale empirique, celui des autorités américaines imbues d'une théorie politique qui fait peu de cas des revendications vincennoises au nom de leur prétendu archaïsme. Ironiquement c'est au prêtre que Turner demande d'américaniser les Français de Vincennes. Mais le Sulpicien fuyant la France révolutionnaire, s'il connait les Lumières, veut-il les appliquer en Amérique?

Cette invite de Turner à se réjouir des bienfaits apportés par les ÉtatsUnis, opposés à l'arbitraire antérieur, doit en fait se lire en regard de ce qui concrètement se trame au même moment à Vincennes. Du passage de Levadoux en 1793 alors que le titulaire à Vincennes est bien Flaget subsiste un procès-verbal:

L'an mille sept cent quatre vingt treize et le vingt huit avril monsieur Michel Levadoux, grand vicaire de monseigneur l'évêque de Baltimore et supérieur de la mission des prêtres de saint sulpice desservant les cures des Illinois et du poste Vincennes étant actuellement au poste Vincennes et ayant convoqué aux prières du prône une assemblée générale pour être tenue à l'issue de la grand messe dans la maison destinée à servir de résidence à monsieur le curé, l'assemblée étant formée de la majeure [partie] des habitants; mr le grand vicaire leur a proposé s'il ne seroit pas plus apropos que l'assemblée nomma cinq syndics avec pleins pouvoirs de la [part] des habitans pour traiter et convenir avec lui sur le traitement que les habitans devoient à Mr le curé et sur lequel conviendroit d'établir ou de Réformer pour la décence du culte:

Cette proposition ayant été acceptée d'une voix unanime, on a procédé en conséquence ou de suite à la nomination des cinq syndics:

MM Guillaume Paget, Louis Deline, Pierre Mallet, Joseph Tougat et Jacques Latrimouille ayant la majorité des vois et ayant accepté la commission, l'as-

62. Archives de l'Université Notre Dame, MAVI 25, George Turner à Benoît-Joseph Flaget, 18 août 1794. C'est Turner qui souligne. 
semblé a été dissoute et monsieur le grand vicaire en a provoqué une nouvelle pour le lendemain avec les susdites syndics.

Le 29 avril 1793 monsieur le grand vicaire et les syndics nommés par la majorité des habitans du Poste Vincennes sont convenus des articles suivants:

1 que MM les syndics mettroient Monsr le curé en possession d'un terrain situé près de l'église le long de la rivière, et se chargeoient de faire toutes les poursuites pour et contre au nom des habitans contre quiconque pourroit y mettre opposition.

2 que monsieur le curé demeureroit seul en possession de la terre de l'église pour en retirer à son seul profit tous les fruits sans être obligé d'en rendre aucun comte.

3 que chaque habitant lui payera, à commence de cette année le vingt-sixième boisseau du produit de toutes les semences qui seront faites, porté et livré dans son grenier, ou la treizième gerbe prise dans les champs à l'option du curé.

4 que chaque habitant lui conduira dans la cour un voyage de bois de chauffage.

5 de Battir, ou achetter, au ou moins louer aux frais des paroissiens une maison et un jardin clos convenable pour la résidence du curé ainsi que de l'entretien et réparacion nécessaires aux sus dits objets.

6 de payer à Mr le curé par tous les habitans le casuel selon l'ancien tarif qui se trouve dans le registre des marguilliers.

7 que [les syndic soient chargés de faire appliquer les articles].

Levadoux, vic et supérieur de la mission

$[\text { marques des syndics }]^{63}$.

Levadoux met en place une véritable restauration. Tous les catholiques sont convoqués afin d'affirmer le rétablissement de l'ordre et de désigner des syndics. Ce sont eux qui doivent accepter des conditions simples qui ont toutes un même objet: le prêtre doit être logé et nourri par les paroissiens et doit être au-dessus d'eux, selon les préceptes tridentins. L'impression que Levadoux impose son autorité sans coup férir doit être nuancée par l'affaire de la dîme. Celle-ci est fixée au $26^{\text {e }}$, ce qui correspond à la norme 
canadienne $^{64}$ à laquelle les Français de Vincennes sont demeurés attachés et qu'ils ont donc su imposer. Il n'y a pas trace ici de négociation, mais en 1797 les habitants du village considèrent avec Rivet qu'il est inutile de nommer de nouveaux syndics tant l'application du taux est "normale $\mathrm{n}^{65}$ », et en 1799, le nouveau prêtre de Cahokia, Ollivier, un Français réfractaire lui aussi, se voit imposer de même la dîme au $26^{\mathrm{e} 66}$.

Il s'agit en fait à Vincennes, et dans les autres postes pourvus ensuite, d'une sorte de double restauration: celle d'un ordre français par des prêtres réfractaires très attachés à un modèle autoritaire tridentin marqué par une culture politique d'Ancien Régime, et celle d'une identité canadienne, ces paroissiens parvenant à imposer leur choix dans une certaine mesure. Or ce phénomène de réaffirmation identitaire intervient au moment où l'intégration aux États-Unis apporte un autre modèle. Faut-il y voir une réaction face à la conquête? Cette réaction étant le fruit d'une intervention extérieure, celle des prêtres français, elle est sujette à caution. Elle aurait tendance à signaler une sorte de repli sur soi des Français de Vincennes, alors que la communauté demeure ouverte et dynamique. On assiste plutôt à un dialogue, malaisé sans doute, entre un clergé soudainement présent et rigoriste et des paroissiens à la fois jaloux de leur identité locale et de leur indépendance et anxieux pour une part de retrouver une certaine normalité catholique. Le contrôle social peut se négocier dans une certaine mesure, du temps de Flaget et Levadoux comme du temps de Gibault.

\section{CONCLUSION}

L'étude du fait religieux, qu'il faut ajouter à la trilogie des enjeux telle qu'elle est définie par Eric Hinderaker - la terre, le commerce, la liberté ${ }^{67}$ - éclaire l'histoire plus globale de Vincennes en ces temps incertains. Alors qu’en 1795 le Traité de Greenville entérine la défaite des Miamis de Little Turtle/Petite Tortue et que celui de Jay repousse les Britanniques au-delà de Détroit, et que donc l'ordre américain semble devoir s'installer enfin avec fermeté (il faudra tout de même attendre 1800 pour voir la création

64. Jean Roy, «Soutenir le prêtre. La dîme comme prélèvement ecclésiastique», dans Serge Courville et Normand Séguin, dir. Atlas historique du Québec. La paroisse (Sainte-Foy, Les Presses de l'Université Laval, 2001), 176-189.

65. Archives du diocèse de Covington, lettres de Jean-François Rivet à $\mathrm{M}^{\mathrm{gr}}$ Carroll, 14 mai 1797.

66. Archives du diocèse de Covington, lettres de Jean-François Rivet à $\mathrm{M}^{\mathrm{gr}}$ Carroll, 18 septembre 1799.

67. Eric Hinderaker, The Elusive Empire..., op. cit. 
d'un Territoire de l'Indiana avec pour capitale Vincennes), les Français de Vincennes sont dans un entre-deux inconfortable. Ils participent à l'évolution de leur monde, ne renoncent pas à leur dynamisme, s'intègrent tant bien que mal aux structures politiques qui les encadrent, mais en même temps ne peuvent que constater l'écart entre leur établissement et l'afflux de colons anglo-américains, et donc leur marginalisation à terme.

Néanmoins en 1795 celle-ci n'est pas achevée, et le Vincennes carrefour, tel qu'il existe depuis sa fondation, n'est pas mort, loin de là. Les incertitudes, qui sont autant de facettes d'un monde colonial complexe, durent encore quelques décennies. Ce n'est que dans les années 1830 que l’Église parvient à contrôler la paroisse dans le sens plein du terme, lorsqu'un évêque, $\mathrm{M}^{\mathrm{gr}}$ Bruté de Rémur, venu de France encore, s’installe. Mais alors Vincennes est devenu une communauté bien marginale: de capitale de l'Indiana elle est devenue centre local sans grande importance. Les chronologies religieuse et politique se sont de nouveau croisées. 